\title{
Effect of Solvents on the Electronic Properties of Fullerene Based Systems: Molecular Modelling
}

\author{
Abdel-Baset H Mekky ${ }^{1 *}$, Hanan G Elhaes ${ }^{2}$, Mohamed M. El-Okr ${ }^{3}$, Abdulaziz S Al-Aboodi ${ }^{1}$ and Medhat A. Ibrahim ${ }^{4}$ \\ ${ }^{1}$ Buraydah Colleges, Al-Qassim, King of Saudi Arabia \\ ${ }^{2}$ Faculty of Women for Arts, Science, and Education, Ain Shams University, Egypt \\ ${ }^{3}$ Faculty Science, El Azhar University, Egypt \\ ${ }^{4}$ Spectroscopy Department, National Research Centre, Egypt
}

\begin{abstract}
Density functional theory (DFT) was utilized as a backup for the experimental study. Solvent effects on the molecular structure and electronic properties of fullerene $\mathrm{C} 60$ and its derivatives were estimated by performing the DFT with B3LYP/3-21G** calculations using the polarized continuum model (PCM) developed by Tomasi. Overall, different forms of quantum-chemistry were used based on performed calculations, including dipole moments (total dipole moment, X, Y, and Z components) and finally, orbital energies, EHOMO, ELUMO; HL gap (gap between EHOMO and ELUMO).
\end{abstract}

Keywords: Computational methods; Fullerene; Solubility; DFT; polarized continuum model; Molecular modeling; Electronic properties

\section{Introduction}

Fullerenes are allotropes of carbon and possess various novel properties that make them useful in the area of nanotechnology and pharmaceuticals. Fullerenes are currently being widely investigated and have potential for various technical applications [1]. In particular, for biomedical testing, water-soluble forms of fullerenes are of outstanding interest.

Numerous efforts have been formed to explain the trends in room-temperature solubility of the fullerenes [2-4]. There are many different approaches to calculate and predict C60 solubility in organic solvents. Some of them are fully mechanistic [5-9], developed from the thermodynamical point of view; others are statistically based, with good correlation coefficients, but not transparent and complicated in interpretation [10-16].

In this work, we aspired to discover simple, transparent relationship and computationally fast approach to predict the solubility of C60 in various solvents. The exact description of solvation phenomena presents a substantial challenge to theoretical chemistry. Two typical approaches are employed to contain the solvent effects. One is the socalled supermolecular approach or the discrete model, where explicit solvent molecules are added and treated at the same level of theory as that employed for the solute. Nevertheless, this supermolecular approach does come with a significant increase in computational expense. Some other approach is the polarizable continuum model (PCM).

Previously, the structures of Fullerenes $c 60$ had been studied in the gas phase by means of the $\mathrm{B} 3 \mathrm{LYP} / 3-21 \mathrm{G}^{* *}$ level of calculation $[17,18]$. We made geometry optimization in vacuo, and the calculation was based along the gas-phase optimized geometries. The solvent effects were identified through the polarizable continuum model (PCM) of Tomasi et al. [19] because the PCM model offers a balanced and theoretically sound treatment of all solute-solvent interactions at a very reasonable computational cost.

\section{Computational Methods}

All the calculations use the polarizable continuum model (PCM) were utilized to describe the thermodynamics of solvation of the fullerenes $\mathrm{C}_{60}$ and its derivatives in some solvents. Solvent effects were evaluated using the (Gaussian $98 \mathrm{w}$ ) [20] implementation of the thermodynamically based polarized continuum model (PCM)' the level of calculations is b3lyp/3-21g** for the ground state.

\section{Results and Discussions}

\section{Solubility of fullerene $\mathrm{C}_{60}$}

The bond lengths and bond angles of the studied compound, $\mathrm{C}_{60}$, were optimized in the gas phase, water, Methanol, Heptane, Acetonitrile, Toluene and Pyrrolidine. Table 1 establishes the important changes of bond length and bond angle for C60 from gas to solve.

a) Minimal value of bond length between carbon atoms;

b) Maximal value of bond length between carbon atoms

c) Minimal value of bond angle between carbon atoms;

d) Maximal value of bond angle between carbon atoms

From Table 1, we can see little changes in the bond lengths of C60 when C60 was optimized with water, Methanol, Heptane, Acetonitrile and Toluene and compared within the gas phase. The great change of bond length and angle value of $\mathrm{C}_{60}$ in the Pyrrolidine compared within the gas phase, water, Methanol, Heptane, Acetonitrile and Toluene. The same bond length and angle in water, Heptane, Acetonitrile and Toluene (slight modifications in the attachment angle of C60 in Methanol).

*Corresponding author: Abdel-Baset $\mathrm{H}$ Mekky, Buraydah Colleges, Saudi Arabia Tel: 0922-5291501-502; E-mail: hofny_a@yahoo.com

Received October 14, 2014; Accepted January 29, 2015; Published February 10,2015

Citation: Mekky ABH, Elhaes HG, El-Okr MM, Al-Aboodi AS, Ibrahim MA (2015) Effect of Solvents on the Electronic Properties of Fullerene Based Systems: Molecular Modelling. J Appl Computat Math 4: 203. doi:10.4172/21689679.1000203

Copyright: @ 2015 Mekky ABH, et al. This is an open-access article distributed under the terms of the Creative Commons Attribution License, which permits unrestricted use, distribution, and reproduction in any medium, provided the original author and source are credited. 


\begin{tabular}{|c|c|c|c|c|}
\hline \multirow{2}{*}{ Solvents } & \multicolumn{4}{|c|}{ Geometry Optimization } \\
\hline & $C-C^{\text {a) }}$ & $C-C^{b)}$ & $\mathrm{C}-\mathrm{C}-\mathrm{C}^{\mathrm{c})}$ & $C-C-C$ d) \\
\hline Gas phase & 1.385 & 1.464 & 108 & 120 \\
\hline Water & 1.389 & 1.461 & 108 & 120 \\
\hline Methanol & 1.393 & 1.457 & 107.9 & 120.2 \\
\hline Heptane & 1.389 & 1.461 & 108 & 120 \\
\hline Acetonitrile & 1.389 & 1.461 & 108 & 120 \\
\hline Toluene & 1.389 & 1.461 & 108 & 120 \\
\hline Pyrrolidine & 1.011 & 1.571 & 100.7 & 124.6 \\
\hline
\end{tabular}

Table 1: Selected structural parameters of the optimized $C_{00}$ in gas and different solvents, bond distance $\left(\mathrm{A}^{\circ}\right)$ and bond angles $\left({ }^{\circ}\right)$. Obtained by the B3LYP methods with $3-21 \mathrm{G}^{* *}$ basis set method which described through the polarizable continuum model (PCM).

\begin{tabular}{|l|c|c|c|}
\hline \multirow{2}{*}{ Solvent } & & & Energy Gap (ev) \\
\cline { 2 - 4 } & HOMO & LUMO & 3.007 \\
\hline Gas phase & -6.531 & -3.523 & 3.007 \\
\hline Water & -6.531 & -3.523 & 3.007 \\
\hline Methanol & -6.531 & -3.523 & 3.007 \\
\hline Heptane & -6.531 & -3.523 & 3.007 \\
\hline Acetonitrile & -6.531 & -3.523 & 3.007 \\
\hline Toluene & -6.531 & -3.523 & 2.7 \\
\hline Pyrrolidine & -6.06 & -3.359 & \\
\hline
\end{tabular}

Table 2: Calculated HOMO/LUMO energy gap as (ev) for $\mathrm{C}_{60}$, which described through the polarizable continuum model (PCM) and calculated by B3LYP methods with $3-21 \mathrm{G}^{* *}$ basis set.

Also, Table 1 shows a slight lengthening of the maximum bond length and angle of $\mathrm{C}_{60}$ in Pyrrolidine (a widening of the maximum bond length and angle), and a narrowing of minimum bond length and angle. Our simulation predicts a lengthening of the max $\mathrm{C}-\mathrm{C}$ bond and the max. C-C-C angle ( $\approx+0.11 \AA$ and 4.6 degrees, respectively) and a narrowing of the min. bond length and angle $(\approx-0.873 \AA$ and 7.3 degrees, respectively), whereas the Methanol-solvent results predict smaller changes for the bond lengths and angles.

The solvents (water, Methanol, Heptane, Acetonitrile and Toluene) effect has a minor influence on these geometrical parameters, the calculations do not evidence any significative effect on the lengths and angles, the only observable change is of $\mathrm{C}_{60}$ in the Pyrrolidine.

The max. C-C bond length in solvent (Pyrrolidine) is longer than that in a vacuum, and the solvents (water, Methanol, Heptane, Acetonitrile and Toluene), these results indicate that the solvent (Pyrrolidine) can reduce the intensity of the max. C-C bond, which might imply that the solvent (Pyrrolidine) is in favor of the $\mathrm{C}-\mathrm{C}$ activation and improve the reaction activity.

Table 2, summarizes the highest occupied molecular orbital (HOMO), the lowest unoccupied molecular orbital (LUMO) and HOMO and LUMO energy gaps for $\mathrm{C}_{60}$ calculated at the DFT level in the $3-21 G^{* *}$ basis set. The values of LUMO and HOMO and their energy gap reflect the chemical activity of the molecule. LUMO as an electron acceptor represents the ability to obtain an electron, while $\mathrm{HOMO}$ as an electron donor represents the ability to donate an electron. The smaller the LUMO and HOMO energy gaps, the easier it is for the HOMO electrons to be excited; the higher the HOMO energies, the easier it is for HOMO to donate electrons; the lower the LUMO energies, the easier it is for LUMO to accept electrons. From the resulting data shown in Table 2, it is obvious that the LUMO energy of $\mathrm{C}_{60}$ in the Pyrrolidine is lower than those of $\mathrm{C}_{60}$ in the gas phase and also, the energy gap of $\mathrm{C}_{60}$ in the Pyrrolidine is smaller than that of $\mathrm{C}_{60}$ in the gas phase. Consequently, the electrons transfer from $\mathrm{HOMO}$ to
LUMO in $\mathrm{C}_{60}$ in the gas phase is relatively easier than that in $\mathrm{C}_{60}$ in the Pyrrolidine. With the decrease of the LUMO energies, LUMO in $\mathrm{C}_{60}$ in the gas phase accepts electrons easily. Soft molecules have a small energy gap.

From Table 2, one can see that the solvent sequence with low to high $\mathrm{HOMO} / \mathrm{LUMO}$ gap (energy gap of $\mathrm{C}_{60}$ in Pyrrolidine<energy gap of $\mathrm{C}_{60}$ in the gas phase, water, Methanol, Heptane, Acetonitrile and Toluene solutions).

The HOMO-LUMO gaps had been studied in the gas phase, water, Methanol, Heptane, Acetonitrile and Toluene solutions, show no effect and the energy gap remains the same. Based on the above description, it is easy to understand why the water, Methanol, Heptane, Acetonitrile and Toluene solutions are suspension of $\mathrm{C}_{60}$.

The results reported in Table 3, show that $\mathrm{C}_{60}$ in the gas phase, water, Methanol, Heptane, Acetonitrile and Toluene solvents possess the lowest dipole moment compared with $\mathrm{C}_{60}$ in Pyrrolidine solvent.

By knowing the value of dipole moment we can conclude that the clusters having a higher dipole moment can be treated as a good solvent.

As expected, the molecule becomes more polarized in the presence of the Pyrrolidine solvent (Table 3), and the molecular dipole moment increases with solvent polarity.

\section{Solubility of specific fullerene derivatives}

The optimized structures of the $\mathrm{C}_{59} \mathrm{X}(\mathrm{X}=\mathrm{C}, \mathrm{B}, \mathrm{Al}, \mathrm{Ga}, \mathrm{In}, \mathrm{N}, \mathrm{P}, \mathrm{As}$, $\mathrm{Sb}$ ) cages in pyrrolidine solvent, the bond lengths and bond angles in pyrrolidine solvent are listed in Table 4 . From Table 4, we can see that all the doped cages in pyrrolidine solvent undergo some distortions due to the dopant atoms, though they still preserve closed cage structures.

a) Minimal value of bond length between carbon atoms;

b) Maximal value of bond length between carbon atoms

c) Minimal value of bond angle between carbon atoms;

d) Maximal value of bond angle between carbon atoms

It is well-known that there are two kinds of $\mathrm{C}-\mathrm{C}$ bond in $\mathrm{C}_{60}$ cage, the [6] bond and the [5,6] bond. In pyrrolidine solvent, The bond lengths are 1.011 and $1.571 \AA$ for [6] and [5,6] bonds, respectively based on our DFT calculations. When the carbon cage is doped by the dopant atom, the $\mathrm{C}-\mathrm{X}$ bonds are presented. From Table 4, it can be determined that the $\mathrm{C}-\mathrm{X}$ bond lengths are in the orbit of 1.011 to $2.143 \AA$. The bond lengths increased obviously for $\mathrm{X}=\mathrm{B}, \mathrm{Al}, \mathrm{Ga}$ and $\mathrm{In}$, ranging from $1.087 \AA$ to $2.143 \AA$ and for $\mathrm{X}=\mathrm{P}, \mathrm{As}$, and $\mathrm{Sb}$, ranging from $1.087 \AA$ to $2.013 \AA$. Nevertheless, the $\mathrm{C}-\mathrm{N}$ bonds in $\mathrm{C} 59 \mathrm{~N}$ are 1.084 and $1.554 \AA$, and therefore the original $[5,6]$ bond (max. bond length) is even decreased by $0.017 \AA$ compared with that in the pristine cage. It is also found that the $\mathrm{C}-\mathrm{X}$ bond lengths increase more significantly for the larger dopant atoms.

For example, the $\mathrm{C}-\mathrm{B}$ bonds are 1.087 and $1.603 \AA$, while the $\mathrm{C}-\mathrm{X}$ bonds $(\mathrm{X}=\mathrm{P}, \mathrm{As}, \mathrm{Sb})$ are estimated to be within 1.087-2.013 . Hence it is clearly that the cage with larger dopant atom gives more obvious distortion.

The bond angles are also listed in Table 4, for fullerene and substituted fullerene in pyrrolidine solvent. From the table it is found that the bond angles are about unchanged for doping with the atoms compared with that of the pristine cage. 


\begin{tabular}{|l|c|c|c|c|c|c|}
\hline Dipole moment contribution & Gas phase & Water & Methanol & Heptane & Acetonitrile & Toluene \\
\cline { 1 - 5 } as Debye & 0 & 0 & -0.0002 & 0 & 0 \\
\hline X & 0 & 0 & 0.0004 & 0 & 0 \\
\hline Y & 0 & 0 & -0.0004 & 0 & 0 \\
\hline Z & 0.0001 & 0 & 0.0006 & 0 & 0 \\
\hline Total dipole moment & & 0 & 0 \\
\hline
\end{tabular}

Table 3: Calculated dipole moment as Debye for $\mathrm{C}_{60}$, which described through the polarizable continuum model (PCM) and calculated by B3LYP methods with 3-21G** basis set.

\begin{tabular}{|l|c|c|c|c|}
\hline \multirow{2}{*}{ Clusters } & \multicolumn{4}{|c|}{ Geometry Optimization } \\
\cline { 2 - 5 } & $\mathbf{C}-\mathbf{C}^{\text {a) }}$ & $\mathbf{C}-\mathbf{C}^{\mathbf{b}}$ & $\mathbf{C}-\mathbf{C}-\mathbf{C}^{\mathbf{c}}$ & $\mathbf{C}-\mathbf{C}-\mathbf{C}^{\text {d) }}$ \\
\hline C60 & 1.011 & 1.571 & 100.7 & 124.6 \\
\hline C59-B & 1.087 & 1.603 & 100.7 & 126.8 \\
\hline C59-Al & 1.089 & 1.828 & 100.8 & 127.9 \\
\hline C59-Ga & 1.089 & 1.924 & 100.8 & 124.6 \\
\hline C59-In & 1.09 & 2.143 & 100.8 & 124.6 \\
\hline C59-N & 1.084 & 1.554 & 100.4 & 127.7 \\
\hline C59-P & 1.087 & 1.64 & 100.7 & 128.1 \\
\hline C59-As & 1.084 & 1.795 & 100.8 & 127.6 \\
\hline C59-Sb & 1.085 & 2.013 & 100.8 & 127.6 \\
\hline
\end{tabular}

Table 4: Calculated bond lengths $(\AA)$ and bond angles $\left({ }^{\circ}\right)$ for $C_{60}$ and some substituted fullerenes, which described through the polarizable continuum mode (PCM) by using Pyrrolidine and calculated by B3LYP methods with $3-21 \mathrm{G}^{\star *}$ basis set.

\begin{tabular}{|l|c|c|c|}
\hline \multirow{2}{*}{ Clusters } & & & Energy Gap (ev) \\
\cline { 2 - 4 } & HOMO & LUMO & 2.7 \\
\hline C60 & -6.06 & -3.359 & 2.72 \\
\hline C59-B & -6.261 & -3.54 & 2.702 \\
\hline C59-Ga & -6.079 & -3.376 & 2.705 \\
\hline C59-In & -6.077 & -3.372 & 2.717 \\
\hline C59-N & -6.127 & -3.41 & 1.7 \\
\hline C59-P & -5.258 & -3.557 & 1.135 \\
\hline C59- As & -4.68 & -3.544 & 1.275 \\
\hline C59- Sb & -4.765 & -3.489 & 1.064 \\
\hline
\end{tabular}

Table 5: Calculated HOMO/LUMO energy gap as (ev) for C60 and some substituted fullerenes, which described through the polarizable continuum model (PCM) by using Pyrrolidine and calculated by B3LYP methods with $3-21 \mathrm{G}^{\star *}$ basis set.

It is well-known that the frontier orbitals, the highest occupied molecular orbital (HOMO) and the lowest unoccupied molecular orbital (LUMO), act as an important role in chemical reaction for the reactant molecule, so the frontier orbital analysis of the doped cages is necessary. In Table 5, the HOMO and LUMO energy levels of the substituted fullerene were summarized. It can be seen that HOMO levels of the doped (for $\mathrm{X}=\mathrm{B}, \mathrm{Al}, \mathrm{Ga}$ and $\mathrm{In}$ ), are all decreased and for doped by $\mathrm{N}, \mathrm{P}, \mathrm{As}$ and $\mathrm{Sb}$ are increased compared with that of $\mathrm{C}_{60}$ As for LUMO levels of the doped cages are all decreased compared with that of $\mathrm{C}_{60}$. As for LUMO levels are increased when doping with $\mathrm{N}, \mathrm{P}$, As and $\mathrm{Sb}$ atoms, compared with that of the pristine cage.

It is recognized that both the thermodynamic stability and kinetic stability have crucial influence on the relative abundances of different fullerene structures. It has been pointed out that higher kinetic stability is usually associated with a larger HOMO-LUMO energy gap, because exciting electrons from a low HOMO to a high LUMO is energetically unfavorable, which would be necessary to activate a reaction. The calculated Eg of the doped cages is listed in Table 5. It can be found that all the doped cages by N, P, As and Sb present smaller Eg than that of $\mathrm{C}_{60}$ cage. So the kinetic stability of the cage is decreased by substitution from viewpoint of HOMO-LUMO gap. And also, It can be found that all the doped cages by B, Al, Ga and In present greater Eg than that of

\begin{tabular}{|c|c|c|c|c|}
\hline Clusters & $\mathbf{X}$ & $\mathbf{Y}$ & $\mathbf{Z}$ & Total dipole moment \\
\hline $\mathrm{C}_{60}$ & 1.9692 & 0 & -1.2089 & 2.3106 \\
\hline $\mathrm{C}_{59} \mathrm{~B}$ & -0.7839 & -0.0001 & -2.5694 & 2.6863 \\
\hline $\mathrm{C}_{59} \mathrm{Al}$ & -2.3374 & 0.0004 & -1.3346 & 2.6916 \\
\hline $\mathrm{C}_{59} \mathrm{Ga}$ & 2.6356 & -1.0881 & 0.0002 & 2.8514 \\
\hline $\mathrm{C}_{59} \mathrm{In}$ & 3.0786 & 0.7356 & 0 & 3.1653 \\
\hline $\mathrm{C}_{59} \mathrm{~N}$ & 0.3106 & 0 & -1.6342 & 1.6635 \\
\hline $\mathrm{C}_{59} \mathrm{P}$ & 0.4219 & 0 & -1.8398 & 1.8875 \\
\hline $\mathrm{C}_{59} \mathrm{As}$ & 1.0084 & 0.0011 & -1.6833 & 1.9622 \\
\hline $\mathrm{C}_{59} \mathrm{Sb}$ & -1.5629 & -1.538 & 0.0011 & 2.1928 \\
\hline
\end{tabular}

Table 6: Calculated dipole moment as Debye for $C_{60}$ and some substituted fullerenes, which described through the polarizable continuum model (PCM) by using Pyrrolidine and calculated by B3LYP methods with $3-21 G^{* *}$ basis set.

$\mathrm{C}_{60}$ cage. So the kinetic stability of the cage is increased by substitution from viewpoint of HOMO-LUMO gap.

Lastly, we have also worked out the dipole moments of the C60 and some substituted fullerenes, which described through the polarizable continuum model (PCM) by using Pyrrolidine and calculated by B3LYP methods with $3-21 \mathrm{G}^{\star *}$ basis set (Table 6).

The dipole moment is defined as the first derivative of the energy with respect to an electric field. The calculated dipole moment can be conveyed in terms of vector in three directions $\mathrm{X}, \mathrm{Y}$ and $\mathrm{Z}$ as indicated in Table 6. The negative mark of both $\mathrm{X}, \mathrm{Y}$ and $\mathrm{Z}$-axis indicates that the dipole moment is pointing away from $\mathrm{X}, \mathrm{Y}$ and $\mathrm{Z}$ direction, while the plus sign of $\mathrm{X}, \mathrm{Y}$ and $\mathrm{Z}$-axis indicates that it points in $\mathrm{X}, \mathrm{Y}$ and $\mathrm{Z}$ direction. Regarding Table 5, one can conclude that, the total dipole moment and the net contribution of charge in the directions $\mathrm{X}, \mathrm{Y}$ and $\mathrm{Z}$ are completely exchanged.

As listed in Table 5 the dipole moment is computed. Regarding $\mathrm{C}_{60}$ the total dipole moment is 2.3106 Debye distributed as $1.9692,-0.0000$ and -1.2089 contributions in the $\mathrm{x}, \mathrm{y}$, and $\mathrm{z}$-directions, respectively. As the effect of doping the change in the dipole moment of $\mathrm{C}_{60}$ and $\mathrm{C}_{59} \mathrm{X}$ was being discussed. The value of dipole moment is increased for $\mathrm{C}_{59} \mathrm{X}$ $(\mathrm{X}=\mathrm{B}, \mathrm{Al}, \mathrm{Ga}, \mathrm{In})$ and it is increased as compared with that of $\mathrm{C}_{6} . \mathrm{An}_{59}$ also, The value of dipole moment is increased for $\mathrm{C}_{59} \mathrm{X}(\mathrm{X}=\mathrm{N}, \mathrm{P}, \mathrm{As}, \mathrm{Sb})$ and it is decreased as compared with that of $\mathrm{C}_{60}$.

The C60 and doped-C60 indicate that doping has an outcome on the dipole moment, which can cause subsequent changes in the electrical properties of the molecular species.

\section{Conclusions}

The solubility of $\mathrm{C} 60$ in the solvents is important to enable purification and chemical change. In universal, the solubility in the majority of solvents is very depressed, because C60 exhibit a high tendency for aggregation. On the other hand the interaction between the solvent molecules and C60 is usually very light, since the fullerene is a nonpolar molecule, which is hardly polarizable due to the large HOMO-LUMO gap. In summary, the solubility of C60 in polar solvents 
Citation: Mekky ABH, Elhaes HG, El-Okr MM, Al-Aboodi AS, Ibrahim MA (2015) Effect of Solvents on the Electronic Properties of Fullerene Based Systems: Molecular Modelling. J Appl Computat Math 4: 203. doi:10.4172/2168-9679.1000203

such as methanol and water is about zero. This low solubility can also be considered in the case of, Heptane, Acetonitrile and Toluene as solvents. The best solubilities are obtained in Pyrrolidine, which makes these solvents to the standard solvents for preparative use.

\section{References}

1. Dresselhaus MS, Dresselhaus G, Eklund PC (1996) Science of Fullerenes and Carbon Nanotubes.

2. Beck MT, Mandi G (1997) Fullerene Sci. Technol. 5: 291-310.

3. Marcus Y, Smith A, Korobov M, Mirakyan A, Avramenko NA (2001) Solubility of C60 Fullerene J Phys Chem B 105: 2499-2506.

4. Heymann D (1996) Carbon 3: 627-631.

5. Marcus $Y$ (1997) Solubilities of buckminsterfullerene and sulfur hexafluoride in various solvents. J Phys Chem 101:8617-8623.

6. Abraham MH, Green CE, Acree WE (2000) Correlation and prediction of the solubility of Buckminsterfullerene in organic solvents; estimation of some physicochemical properties. J Chem Soc Perkin Trans 2: 281-286.

7. Hansen CM, Smith AL (2004) Using Hansen solubility parameters to correlate solubility of $\mathrm{C} 60$ fullerene in organic solvents and in polymers. Carbon 42: 1591-1597.

8. Stukalin EB, Korobov MV, Avramenko NV (2003) Solvation free energies of the fullerenes $\mathrm{C} 60$ and $\mathrm{C} 70$ in the framework of polarizable continuum model. J Phys Chem B 107: 9692-9700.

9. Kiss IZ, Mandi G, Beck MT (2000) Artificial neural network approach to predict the solubility of $C 60$ in various solvents. J Phys Chem A 104: 8081-8088.

10. Liu H, Yao X, Zhang R, Liu M, Hu Z (2005) Accurate quantitative structureproperty relationship model to predict the solubility of $\mathrm{C} 60$ in various solvents based on a novel approach using a least-squares support vector machine. J Phys Chem B 109: 20565-20571.
11. Toropov AA, Leszczynska D, Leszczynski J (2007a) QSPR study on solubility of fullerene $\mathrm{C} 60$ in organic solvents using optimal descriptors calculated with SMILES. Chem Phys Lett 441: 119-122.

12. Toropov AA, Leszczynska D, Leszczynski J (2007b) Predicting water solubility and octanol water partition coefficient for carbon nanotubes based on the chiral vector. Comput Biol Chem 31: 127-128.

13. Toropov AA, Rasulev BF, Leszczynska D, Leszczynski J (2007c) Additive SMILES based optimal descriptors: QSPR modeling of fullerene C60 solubility in organic solvents. Chem Phys Lett 444: 209-214.

14. Toropov AA, Rasulev BF, Leszczynska D, Leszczynski J (2008) Multiplicative SMILES-based optimal descriptors: QSPR modeling of fullerene C60 solubility in organic solvents. Chem Phys Lett 457: 332-336.

15. Toropov AA, Toropova AP, Benfenati E, Leszczynska D, Leszczynski J (2009) Additive InChl-based optimal descriptors: QSPR modeling of fullerene C60 solubility in organic solvents. J Math Chem 46: 1232-1251.

16. El-Oker MM, Mekky ABH, Elhaes H, Medhat A (2012) Ibrahim " Electronic Properties of Group V Substituted Fullerene: DFT Approach "International Journal of Scientific \& Engineering Research, 3.

17. Mekky ABH, Elhaes H, El-Oker MM, Ibrahim MA (2012) " Electronic Properties of Substituted C59X (X= B, Al, Ga, In) Fullerene " Materials Science : An Indian Journal 8: 10

18. Tomasi J, Persico M (1994) ' Molecular Interactions in Solution: An Overview of Methods Based on Continuous Distributions of the Solvent '. Chem Rev 94: 2027-2094.

19. Frisch MJ (2001) GAUSSIAN 98 (Revision A 11), Gaussian, Pittsburgh PA.

20. Barone V, Cossi M (1998) 'Quantum Calculation of Molecular Energies and Energy Gradients in Solution by a Conductor Solvent Model', Journal of Physical Chemistry A 102: 1995-2001. 